\title{
Delirium: the role of psychiatry
}

\author{
David Meagher
}

Acute mental disturbance associated with physical illness is well described in early medical literature, but it was not until $1 \mathrm{AD}$ that Celsus coined the term 'delirium' (Lindesay, 1999). Although delirium has many synonyms that are applied in particular clinical settings (Box 1), all acute disturbances of global cognitive functioning are now recognised as 'delirium', a consensus supported by both ICD-10 (World Health Organization, 1992) and DSM-IV (American Psychiatric Association, 1994) classification systems. Delirium is a complex neuropsychiatric syndrome that typically involves a plethora of cognitive and non-cognitive symptoms, resulting in a broad differential diagnosis dominated by mental disorders. Psychiatrists' skills in assessing cognitive function and psychopathology, coupled with their knowledge of psychotropic agents, make them well suited to improving detection, coordinating management and facilitating research into this understudied disorder.

\section{Symptoms of delirium}

The symptoms of delirium include a constellation of physical, biological and psychological disturbances. Impaired attention is considered the core cognitive disturbance and is emphasised in DSMIV and ICD-10 (Table 1). In addition, most patients experience disturbances of memory, orientation, language skills, mood, thinking, perception, motor behaviour and the sleep-wake cycle. Although individual delirium symptoms are non-specific, their pattern is highly characteristic: acute onset (sometimes abruptly, but often over hours or days), fluctuant course (symptoms tend to wax and wane

\section{Box 1 Delirium has many synonyms}

Acute brain failure

Acute confusional state

Acute organic syndrome

Cerebral insufficiency

Encephalopathy

Postoperative psychosis

Toxic psychosis

over any 24-hour period and typically worsen at night) and transient nature (in most cases, delirium resolves within days or weeks) are typical. Delirium also frequently involves a prodromal phase over 23 days of malaise, restlessness, poor concentration, anxiety, irritability, sleep-disturbance and nightmares. A consequence of this broad symptom profile is that delirium has many guises and, depending on prevailing pattern, is easily mistaken for dementia or functional psychiatric disorders (Fig. 1).

\section{Frequency in clinical practice}

Delirium is a common problem in all health care settings, with a point prevalence in general hospital patients of $10-30 \%$. Among the elderly, $10-15 \%$ have delirium on admission and a further $10-40 \%$ develop delirium during the course of their hospital stay (Bucht et al, 1999; Fann, 2000). Rates vary according to the population assessed, study setting and identification methods used, but overall delirium is more frequent in older populations, those

David Meagher was the 1998 Eli-Lilly Royal College of Psychiatrists Travelling Fellow and spent a sabbatical studying the neuropsychiatry of delirium with Paula Trzepacz at the University of Mississippi. He is a consultant psychiatrist in the Midwestern Regional Hospital Limerick (Midwestern Regional Hospital Limerick, Department of Psychiatry, Limerick, Republic of Ireland; e-mail: davidjmeagher@ireland.com) and has ongoing interests in the study of phenomenology and management of delirious patients. 
Table 1 Diagnostic criteria for delirium

ICD-10

Clouding of consciousness, i.e. reduced clarity of awareness of environment, with reduced ability to focus, sustain or shift attention

Disturbed cognition, with impaired immediate recall and recent memory but relatively intact remote recall, and disorientation in time, place or person

At least one of: variable activity levels, increased reaction time, altered flow of speech or enhanced startle reaction

At least one of: insomnia, daytime drowsiness, reversal of sleep-wake cycle, nocturnal worsening of symptoms or disturbing dreams and nightmares

Symptoms are of rapid onset and fluctuate over the course of the day

There is evidence of an underlying cause
$D S M-I V$

Disturbed consciousness, with reduced ability to focus, sustain or shift attention

Altered cognition (memory, orientation, language) or the development of a perceptual disturbance that is not better accounted for by dementia

Disturbance develops over hours to days and tends to fluctuate during the course of the day

There is evidence of an aetiological cause with pre-existing cognitive impairment and certain medical or surgical problems (Box 2). The frequency or relevance of subclinical cases is poorly studied. Rates of delirium are increasing in tandem with the rise in mean age of the general population. It is clear that delirium will assume greater importance as health care systems attempt to provide for our increasingly aged population.

The presence of delirium is not always considered an indication for seeking psychiatric consultation (Francis et al, 1990). Nevertheless, delirium is common in patients referred to consultation-liaison psychiatry services because it is frequently misdiagnosed by referring clinicians. Overall, approximately $10 \%$ of consultation-liaiason referrals have delirium and around $10 \%$ of delirious general hospital patients receive a psychiatric consultation (Sirois,

\section{Box 2 Prevalence of delirium in different populations}

General population: $0.4 \%$

General population ( $>55$ years): $1.1 \%$

General hospital admissions: 9-30\%

Elderly general hospital admissions: $\mathbf{5 - 5 5 \%}$

Elderly accident and emergency attenders: $16 \%$

AIDS: $17-40 \%$

Cancer patients (terminal stages): $25-40 \%$ (28-85\%)

Postoperative patients: $5-75 \%$

Intensive care unit patients: $12-50 \%$

Nursing home residents: up to $60 \%$
1988; Francis et al, 1990), with the involvement of psychiatrists reserved for more complex cases. However, given the frequency of misdiagnosis and the tendency for treatment efforts to focus on underlying cause or behavioural problems rather than severity of actual delirium symptoms (Meagher et al, 1996), more frequent involvement of psychiatrists can improve management of delirium.

\section{Symptom overlap with other disorders}

Changes in mental state can be the most obvious indicator of serious underlying physical illness, especially in the elderly, where delirium is frequently the earliest sign of infection or ischaemic heart disease (Wahlund \& Bjorlin, 1999). The differential diagnosis of delirium is dominated by mental disorders, and accurate detection is further complicated by the fact that delirium frequently coexists with other disorders. Up to two-thirds of cases of delirium occur superimposed on dementia, but the two conditions can usually be distinguished because delirium symptoms tend to dominate the clinical picture (Trzepacz et al, 1998a). Abrupt onset and fluctuating course are typical and highly suggestive of delirium. Moreover, delirium is characterised by marked disturbance of attention, with associated deficits in memory and orientation, disorganised thinking and perceptual disturbance. In contrast, alertness is generally consistent in dementia and thought content is reduced. Delirium should not be mistaken for 'sundowning', which 


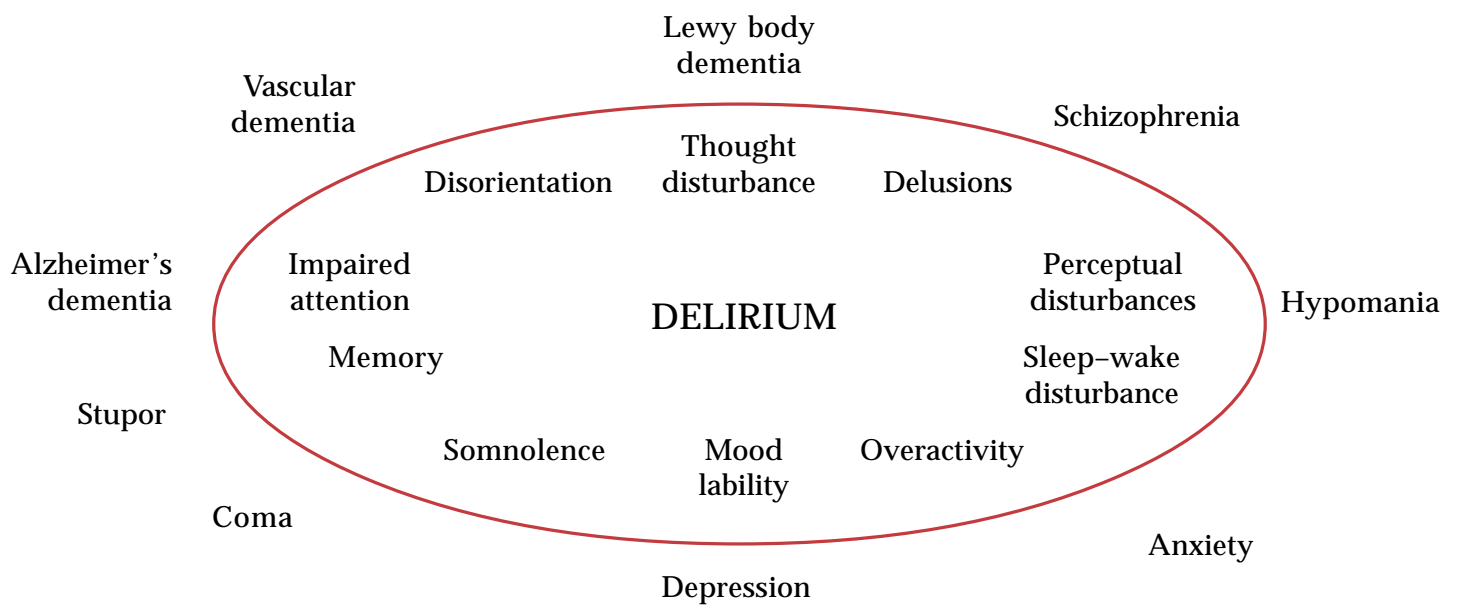

Fig. 1 The differential diagnosis of delirium reflects its broad symptom profile

generally applies to the relatively benign fluctuation in mental state that can occur in dementia - although the relationship between sundowning and delirium requires clarification.

The presentation of delirium can mimic functional psychiatric disorders. Emotional and behavioural changes of delirium are easily mistaken for adjustment reactions, particularly in patients who have experienced major trauma or have cancer. Delirium is frequently confused with depression, especially in females and those with hypoactive or lethargic delirium presentations (Nicholas \& Lindsay, 1995; Armstrong et al, 1997). Most symptoms of major depression can occur in delirium (e.g. psychomotor slowing, sleep disturbances and irritability), but the onset of depressive illness is generally less acute and mood disturbance dominates the clinical picture. Moreover, cognitive impairment in depression typically resembles dementia more closely than delirium - 'depressive pseudodementia'. Hyperactive presentations of delirium can mimic similar disturbances in patients with anxiety disorders, agitated depression or mania. The overlap is further complicated by the fact that delirium can be precipitated by dehydration in patients with severe depression who cannot maintain fluid intake. However, accurate diagnosis of delirium is important because misdiagnosis as depression results in delayed treatment and exposure to antidepressant treatments, many of which have anticholinergic properties that can aggravate delirium.

The disturbances of thought and perception that occur in delirium are generally fluctuant and fragmentary and rarely have the complexity of psychotic symptoms that occur in schizophrenia.
First-rank symptoms are uncommon and hallucinations tend to be visual rather than auditory. Consciousness, attention and memory are generally less impaired in schizophrenia, except in the acute phase of psychosis when marked perplexity can produce a pseudodelirious picture. Delirium involves both qualitative and quantitative alterations in consciousness, and in hypoactive patients can be associated with lethargy, but patients should be rousable. This differs from the marked reduction in consciousness with unrousability that occurs in comatose patients. In children, delirium can present with unexplained behavioural changes, the true nature of which only becomes apparent with close scrutiny of cognitive state.

\section{Underdiagnosis in clinical practice}

Poor recognition remains the single greatest obstacle to improved clinical and research activity in delirium. It is commonplace for delirium to be either missed or recognised late, with over one-third of cases not detected in clinical practice. Identification problems exist across all clinical settings, including general hospital populations, consultation-liaison psychiatry referral samples and even neurologists and psychiatrists providing specialist assessment. Johnson et al (1992) studied consecutive elderly general hospital admissions and noted that delirium was explicitly recognised in 5\% and documented as a synonym in $18 \%$, with variable but poor recognition of individual delirium symptoms as well 
as diagnosis. Missed cases were diagnosed as dementia $(25 \%)$, a functional psychiatric disorder ( $25 \%$ ) or no diagnosis documented $(50 \%)$. Lewis et al (1995), in a study of elderly accident and emergency attenders with delirium, found that a change in mental status was noted in only $13 \%$ and almost a third were discharged directly home. Nonidentification is especially frequent in older patients (where a 'quiet' hypoactive picture is common) and in referrals from surgical wards and intensive care settings (Armstrong et al, 1997). Although some cases are recognised but labelled as one of the many synonyms for delirium, the majority reflect nonidentification of both the individual symptoms and diagnosis of delirium. Explicit recognition of delirium is associated with better outcomes in the form of shorter in-patient stays and lower mortality (Rockwood et al, 1994).

\section{Improving detection}

A classical stratagem of liaison psychiatry involves the brief attachment of a psychiatrist to a medical team, during which time skills of detection, differential diagnosis and treatment are passed on before the psychiatrist moves to another team. This approach is ideal for enhancing delirium detection because non-identification reflects the underappreciation of delirium as a distinct entity and uncertainty regarding definition and diagnostic indicators. The principal diagnostic criteria in ICD10 and DSM-IV are acute onset, fluctuant course and prominent disturbances of attention. Physicians are less familiar than psychiatrists with simple methods of assessing attention, such as serial sevens and digit span, and are more likely to rely on subjective concepts such as 'clouding of consciousness' to identify delirium - an approach that is prone to inconsistency and inaccuracy in diagnosis (O'Keefe \& Gosney, 1997).

Psychiatrists can aid diagnosis by clarifying symptoms, assessing cognitive status and advising on supplementary investigation. Electroencephalography and a range of investigative tools that assess delirium symptoms (see below) can be useful in distinguishing delirium from dementia and functional psychiatric disorders. Delirium identification is improved when cognitive assessment is used routinely and can be enhanced by using simple

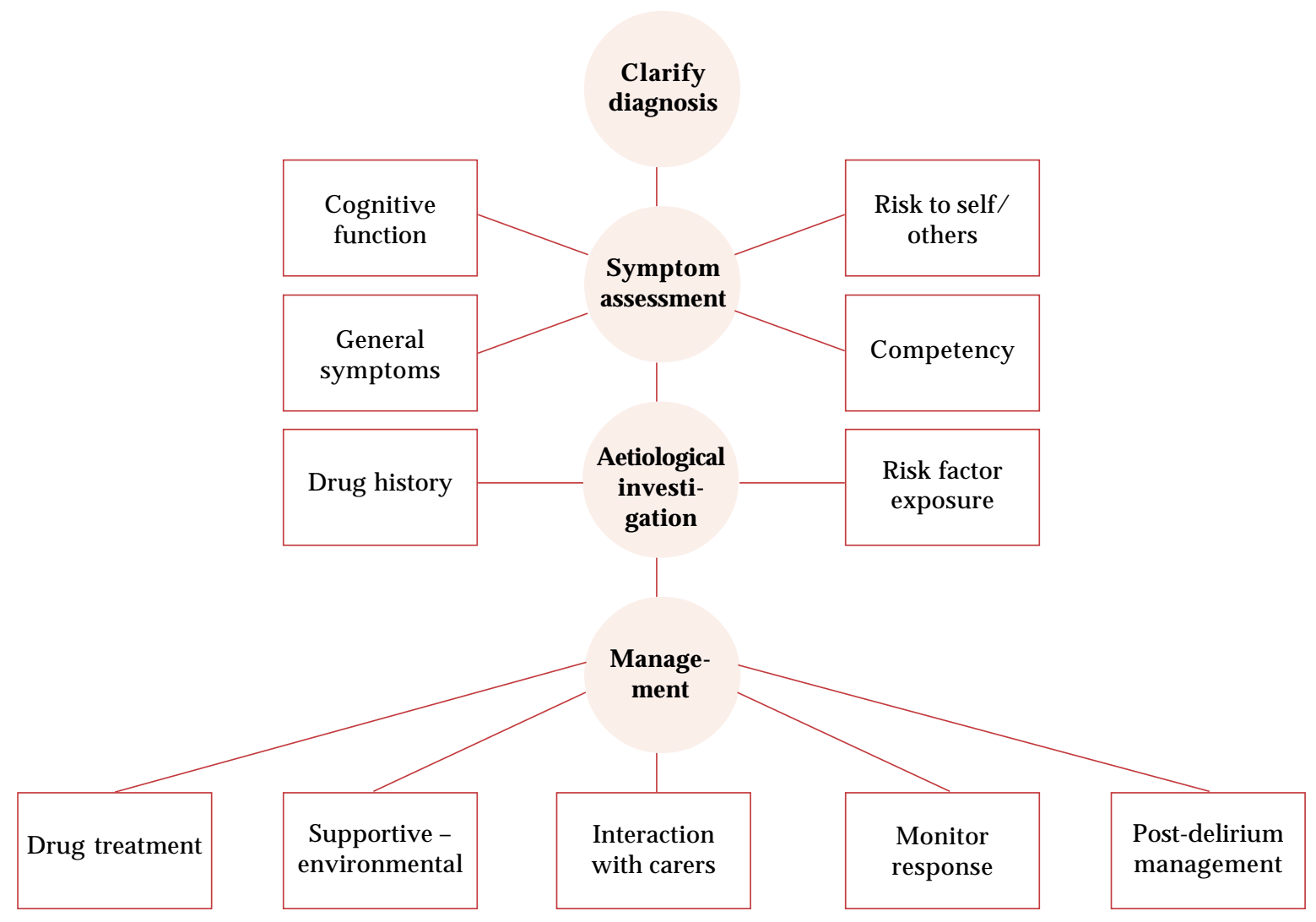

Fig. 2 The role of psychiatrists in delirium management 
screening instruments such as the Confusion Assessment Method (CAM; Inouye et al, 1990). Moreover, interventions aimed at increasing awareness of delirium and encouraging routine cognitive assessment with formal testing can increase detection of delirium and are reflected in improved outcomes (Rockwood, 1999).

\section{Psychiatrists' expertise in delirium management}

Delirium is par excellence a disorder requiring a multifaceted biopsychosocial approach to assessment and treatment. Psychiatrists are thus ideally skilled to coordinate the multi-disciplinary treatment of delirium and can fulfil a range of important functions (Fig. 2). In addition to clarifying the differential diagnosis of patients with suspected delirium, psychiatrists have an important role in the assessment of delirium symptoms and premorbid cognitive status. Psychiatrists are familiar with the problem behaviours that can occur in delirious patients and assessing the risk they pose both to themselves and others. It has been estimated that approximately $7 \%$ of patients attempt some form of deliberate self-harm during a delirium episode (Nicholas \& Lindsay, 1995). Psychiatrists are sometimes asked to assess competency. Given the highly fluctuant nature of cognitive impairment in delirium, which includes periods of relative or absolute lucidity, the ability of patients to participate in decision-making can vary greatly over time, but delirium does not preclude patients from making a useful contribution towards treatment decisions.

Psychiatric consultation facilitates identification of predisposing and precipitating factors for delirium. Medication exposure, visual and hearing impairments, sleep deprivation, uncontrolled pain, dehydration, malnutrition, catheterisation and use of restraints are all factors that can be modified with substantial clinical benefit. Current delirium pharmacotherapies have evolved from use in the treatment of mainstream psychiatric disorders and hence psychiatrists are well acquainted with the practicalities of their use. Medications are implicated as significant contributing factors in over one-third of cases and can act as either protective or risk factors for delirium. Familiarity with their use is a valuable asset in dissecting out their role in any individual case. A range of pre-operative psychological interventions aimed at patient education and anxiety reduction may have preventive value, but these require more explicit study before they warrant introduction into routine clinical practice.
The pharmacological, environmental and psychological management of a delirium episode and its aftermath has been reviewed in detail elsewhere (American Psychiatric Association, 1999; Meagher, 2001). The principles of good ward management of delirious patients include ensuring the safety of the patient and their immediate surroundings, achieving optimal levels of environmental stimulation and minimising the effects of any sensory impediments. Reorientation is facilitated by a predictable environment with clear communication from carers and provision of multiple cues and frequent reminders as to circumstances and setting. The complications of delirium can be minimised by careful attention to the potential for falls and avoiding prolonged hypostasis. Psychiatrists can advise regarding the appropriateness and dosing of drug treatment and help monitor treatment response. Medication use in delirium often represents a response to problem behaviours rather than the severity of actual delirium symptoms. No placebo-controlled trials of pharmacological treatments for delirium have been conducted, but typical antipsychotics, especially haloperidol, are widely used. The available evidence suggests that antipsychotics are effective in alleviating a range of delirium symptoms in patients with either hyperactive or hypoactive clinical profiles (Platt et al, 1994). Moreover, their therapeutic impact is not merely due to their sedative effects and may reflect a specific antidelirium effect, perhaps mediated by effects on the dopamine-acetylcholine balance. Appropriate doses for delirium treatment have not been established, but low-dose haloperidol is appropriate for most patients who require drug treatment. However, caution is required when delirium symptoms occur in the setting of suspected Lewy body dementia, as serious adverse effects can occur with antipsychotic use, and preliminary evidence suggests that alternative strategies such as procholinergic agents may be more appropriate in these cases. A range of other psychotropic agents have been suggested as therapeutic options (e.g. mianserin, trazodone and atypical antipsychotics), but their role and relationship to more standard drug treatments remains to be determined. Benzodiazepine use in delirium requires careful consideration as they are less effective than antipsychotics except in substance/alcohol-related deliria and have the disadvantage of operating as potential aggravating factors in delirium (Breitbart et al, 1996). However, benzodiazepines can be a useful adjunctive treatment in patients prone to adverse effects from antipsychotic agents.

Psychiatrists have the necessary skills to provide supportive psychotherapeutic input and interaction with relatives and carers that is fundamental to good management of delirium. Relatives can play an 
integral role in efforts to support and reorientate delirous patients, but ill-informed, critical or anxious carers can add to the burden of a delirious patient. A therapeutic triangle can emerge whereby medical staff respond to the distress of relatives by medicating patients, which in turn complicates ongoing cognitive assessment. Clarification of the cause and meaning of symptoms combined with recognition of treatment goals can allow better management of what is a distressing experience for both patient and loved ones.

The after-care of delirium has received limited study, but denial, depression and post-traumatic stress disorder are recognised psychological sequelae. Recovered patients are often uncomfortable discussing the experience, but most do recognise its transient nature (Schofield, 1997). Simple clarification can reduce the likelihood of patients or their relatives misinterpreting an episode of delirium as evidence of brain damage or as the first step towards senility or madness. Patients' unwillingness to acknowledge the experience may negatively influence their attitude to help-seeking for medical problems in the future. Inouye et al (1999) have demonstrated the positive impact of a risk factor reduction programme on reducing the number and duration of delirium episodes in hospitalised elderly patients. Explicit recognition of delirium and its associated causes allows minimisation of future exposure to risk factors.

\section{Delirium management: a challenge for psychiatry services}

Delirium management is dictated primarily by the setting in which it occurs. Historically, there has been an overemphasis on identification and treatment of underlying cause without adequate appreciation of the importance of addressing actual delirium symptoms. Delirium is associated with longer hospital stays, reduced independence after discharge and increased mortality that is relatively independent of underlying cause (Francis et al, 1990). Greater recognition of the range of skills required to optimally manage the condition is needed. No single speciality has assumed responsibility for the clinical management or scientific study of delirium, and as a consequence there is a lack of cohesion in the field, reflected in inconsistent nomenclature and variable research methodologies. Traditionally, psychiatrists have contributed substantially to delirium study, but greater interdisciplinary collaboration and more frequent involvement of psychiatry services are needed.

Involvement of psychiatry services tends to occur late in treatment efforts and frequently reflects a desire for advice on placement issues rather than acute treatment. Earlier intervention has many advocates and can positively influence outcome. Referral practices are biased towards hyperactive and disturbed patients, but there is a need for greater emphasis of the less obvious somnolent or hypoactive clinical subtype (Meagher \& Trzepacz, 2000). Although hypoactive patients are perceived as less morbid, they have poorer outcomes that, in part, reflect poorer identification and less aggressive treatment. Moreover, the efficacy of antipsychotics in the treatment of patients with both hypoactive and hyperactive profiles is poorly appreciated (Platt et al, 1994), with much lower utilisation of antipsychotic agents in hypoactive patients (Meagher et al, 1996). Psychiatry services therefore need to be more proactive in identifying hypoactive patients.

Modern consultation-liaiason psychiatry services are often overburdened with the demands posed by functional psychiatric disorders and deliberate selfharm. Although it is difficult to provide delirium assessment in such services, the potential benefits are compelling. Psychiatrists can make valuable contributions at many points along the care pathway for patients with delirium (Fig. 2) and their involvement is associated with clinical improvement in delirium (Hales et al, 1988). In addition, immense financial savings can accrue from reduction of inpatient stays by a single day - a goal that is attainable with systematic treatment interventions (Cole et al, 1994). The shift of in-patient psychiatric care to general hospital settings has made it more feasible for psychiatrists to contribute to the management of delirium and the potential benefits suggest that delirium warrants higher priority as services evolve.

\section{Formal assessment of delirium}

Over the past 20 years, there have been substantial developments in neuropsychology, particularly as it applies to the elderly. One result has been the development of a range of tools for identifying, diagnosing and assessing symptom profile in delirium. Many instruments have been designed for specific purposes and therefore the instrument used in any particular setting needs to be selected carefully. Key questions to be considered include: (1) what is the reason for using the instrument? (2) who will be conducting the assessments and within what time frame? and (3) what is the level of functioning and 
ability to cooperate with assessment procedures within the setting of the population to be studied?

Numerous screening instruments for impaired cognition are available, but a reliable distinction between delirium and dementia requires that the mode of onset and course of symptoms are accounted for. Formal delirium diagnosis requires documentation of acute onset and fluctuant course. As a result, cognitive impairment identified with instruments such as the Mini-Mental State Examination (MMSE; Folstein et al, 1975) must be supplemented by application of DSM or ICD criteria, either by an experienced clinician or with an operationalised instrument (Table 2). The CAM is an operationalisation of key components of DSM-III-R that has high sensitivity and specificity, allows a diagnosis of delirium and is readily incorporated as a screen for delirium into routine clinical settings. However, it has reduced sensitivity when used by nursing staff rather than physicians (see Box 3).

The important attributes of scales for assessing delirium symptom severity are outlined in Table 2 . These instruments generally have good coverage of delirium symptoms and are suitable for serial use. The Delirium Rating Scale (DRS; Trzepacz et al, 1998) is the most widely used to date and, although it requires interpretation by a skilled clinician of information from multiple clinical sources, it has the significant advantage of distinguishing between the disturbances of delirium and dementia. Scores have been shown to predict duration of delirium episode, correlate with frequency of complications and reflect improving clinical state linked to successful drug treatment (Rutherford et al, 1991; Wada \& Yamaguchi, 1993; Nakamura et al, 1997). The revised version (DRS-98; Trzepacz et al, 2000) incorporates more detailed cognitive assessment (including specific items for disturbances of attention, thought processes and language function) and has separate items for both reduced and increased motor activity. Psychometric evaluation is ongoing (Trzepacz et al, 2000).

Many patients with delirium are physically very unwell and their ability to cooperate with detailed assessments of multiple neuropsychological functions is limited. As a consequence, the instruments that have been applied to delirium research have not involved detailed investigation of the range and complexity of neuropsychological disturbances that can occur. The MMSE has been used in many studies but does not distinguish between acute and chronic disturbances, is heavily reliant on subject cooperation (e.g. verbal capacity) and emphasises neuropsychological functions linked to left-cerebral hemispheric activity. Trail-making tests are superior to the MMSE in distinguishing delirium from dementia (Trzepacz et al, 1988b) but lack specificity, and performance is significantly reduced by any condition that affects concentration, motivation or is associated with fatigue. Consequently, they have limited applicability to highly morbid populations with delirium. More recently, it has become increasingly apparent that many of the core disturbances of delirium reflect non-dominant hemispheric functions. Attention, for example, is related to nondominant orbitofrontal, prefrontal and posterior parietal regions. Moreover, recent work suggests that right-sided cognitive functions are especially helpful in distinguishing delirium from dementia and functional psychotic disorders (Hart et al, 1997). The Cognitive Test for Delirium (CTD; Hart et al, 1996) is a recently developed instrument that allows detailed investigation of a range of neuropsychological functions (orientation, comprehension, attention, vigilance and memory) and is suitable for use in

Table 2 Delirium symptom assessment instruments

\begin{tabular}{|c|c|c|c|c|c|}
\hline & Diagnostic & Use & $\begin{array}{l}\text { Symptom } \\
\text { coverage }\end{array}$ & $\begin{array}{l}\text { Distinguishes } \\
\text { delirium from } \\
\text { other disorders }\end{array}$ & $\begin{array}{l}\text { Usefulness in } \\
\text { uncooperative } \\
\text { subjects }\end{array}$ \\
\hline $\begin{array}{l}\text { Delirium Rating } \\
\text { Scale(DRS); } \\
\text { Trzepacz et al, 1998) }\end{array}$ & DSM-III-R & $\begin{array}{l}\text { Trained } \\
\text { clinician }\end{array}$ & Good & Yes & Good \\
\hline $\begin{array}{l}\text { Delirium Symptom } \\
\text { Interview (DSI); } \\
\text { Albert et al, 1992) }\end{array}$ & DSM-III & Lay interpreter & Fair & No & Good \\
\hline $\begin{array}{l}\text { Delirium Assessment } \\
\text { Scale (DAS); } \\
\text { O'Keefe et al, 1994) }\end{array}$ & No & Physician & Good & No & Fair \\
\hline $\begin{array}{l}\text { Confusional State } \\
\text { Evaluation; } \\
\text { (Robertsson, 1999) }\end{array}$ & DSM-III-R & $\begin{array}{l}\text { Doctor, nurse } \\
\text { or psychologist }\end{array}$ & Good & No & Fair \\
\hline
\end{tabular}


Box 3 Confusion Assessment Method (CAM)

Delirium diagnosed if $(a)+(b)+$ one of either (c) or (d):

(a) Acute onset and fluctuating course Evidence of an acute change in mental status from the patient's baseline that changes in severity during the day

(b) Inattention Patient has difficulty focusing attention, e.g. is easily distractible or has difficulty keeping track of conversation

(c) Disorganised thinking

Patient's thinking is disorganised or incoherent, as evidenced by rambling or irrelevant conversation and unclear or illogical flow of ideas

(d) Altered consciousness

A rating of a patient's level of consciousness as other than alert (normal) i.e. vigilant or hyperalert, lethargic or drowsy, stuporous or comatose

patients whose ability to interact with the examiner may be compromised by immobility, intubation or an absence of verbal abilities. This instrument is therefore a significant advance that should allow greater characterisation of the neuropsychological impairments of delirium, their relationship to noncognitive symptoms and a range of other important aspects of clinical profile such as underlying aetiology, treatment responsiveness and course.

\section{Contribution of consultation- liaison psychiatry to delirium research}

The recent publication of treatment guidelines for delirium by the American Psychiatric Association (1999) is an important landmark in delirium research but also serves to highlight many of the shortcomings of the present knowledge base. Much basic information is lacking - opportunities exist for important research that does not require major funding or sophisticated technology but does require careful consideration of methodological issues. These include the methods of identifying study population, accounting for the presence and relevance of concomitant dementia, clearly documenting the character and impact of treatment exposure, and careful selection of suitable tools for measuring symptoms, underlying aetiology, course and outcome. Important information about phenomenology and its relationship to course and outcome is lacking. For example, prospective study is needed of the evolution of symptoms, importance of prodromal features and subclinical cases, factors associated with delirium resolution and the relationship of neuropsychological disturbances to psychopathological symptoms. With the ever-increasing range of psychopharmacological options available for delirium treatment, drug management should be determined according to clinical profile, but there is a need to identify predictors of response to the different therapeutic inputs, including the interaction between pharmacological and environmental therapies. Psychiatrists, with their skills in assessing these various aspects of delirium, can make crucial contributions to furthering our understanding of these important issues.

\section{Conclusions}

Delirium is a complex neuropsychiatric syndrome that is common in all health care settings. The field of delirium is hampered by poor detection - a problem that psychiatrists can assist in reducing both through consultation in complex cases and educational interventions focusing on recognition of key diagnostic indicators and the varying clinical presentations of delirium in clinical practice. Identifying causation, dealing with problem behaviours and treating delirum symptoms and their aftermath present substantial challenges for health care services. Psychiatrists can play a pivotal role in the multifaceted treatment approach that is needed to manage delirious patients. Developments in geriatric neuroscience, specifically in relation to delirium classification and assessment, have reduced the methodological limitations that previously hindered delirium research and herald an exciting period of study. Moreover, the location of modern psychiatry services in community and general hospital settings allows psychiatrists to participate more readily in the treatment and study of this underappreciated complex neuropsychiatric syndrome.

\section{References}

Albert, M. S., Levkoff, S. E., Reilly, C., et al (1992) The Delirium Symptom Interview: an interview for the detection of delirium symptoms in hospitalised patients. Journal of Geriatric Psychiatry and Neurology, 5, 14-21. 
American Psychiatric Association (1994) Diagnostic and Statistical Manual of Mental Disorders (4th edn) (DSM-IV). Washington DC: APA.

* - (1999) Practice Guidelines for the Treatment of Patients with Delirium. Washington, DC: APA.

Armstrong, S. C., Cozza, K. L. \& Watanabe, K. S. (1997) The misdiagnosis of delirium. Psychosomatics, 38, 433-439.

Breitbart, W., Marotta, R., Platt, M. M., et al (1996) A double blind trial of haloperidol, chlorpromazine and lorazepam in the treatment of delirium in hospitalised AIDS patients. American Journal of Psychiatry, 153, 231-237.

Bucht, G., Gustafson, Y. \& Sandberg, O. (1999). Epidemiology of delirium. Dementia and Geriatric Cognitive Disorders, 10, 315-318.

Cole, M. G., Primeau, F. J., Bailey, R. F., et al (1994) Systematic intervention for elderly in-patients with delirium: a randomised trial. Canadian Medical Association Journal, 151, 965-970.

Fann, J. R. (2000) The epidemiology of delirium: a review of studies and methodological issues. Seminars in Clinical Neuropsychiatry, 5, 64-76.

Folstein, M. F., Folstein, S. E., McHugh, P. R. (1975) MiniMental State: a practical method for grading the cognitive state of patients for the clinician. Journal of Psychiatric Research, 12, 189-98.

Francis, J., Martin, D. \& Kapoor, W. N. (1990) A prospective study of delirium in hospitalised elderly. Journal of the American Medical Association, 263, 1097-1101.

Hales, R. E., Polly, S. \& Orman, D. (1988) An evaluation of patients who received an organic mental disorder diagnosis on a psychiatric consultation-liaison service. General Hospital Psychiatry, 11, 88-94.

${ }^{*}$ Hart, R. P., Levenson, J. L., Sessler, C. N., et al (1996) Validation of a cognitive test for delirium in medical ICU patients. Psychosomatics, 37, 533-546.

- Best, A. M., Sessler, C. N., et al (1997) Abbreviated Cognitive Test for delirium. Journal of Psychosomatic Research, 43, 417-423.

Inouye, S. K., van Dyck, C. H., Alessi, C. A., et al (1990) Clarifying confusion: the Confusion Assessment Method a new method for detection of delirium. Annals Internal Medicine, 113, 941-948.

*-, Bogardus, S. T., Charpentier, P. A., et al (1999) A multicomponent intervention to prevent delirium in hospitalized older patients. New England Journal of Medicine, 340, 669-676.

Johnson, J. C., Curse, N. M., Gottlieb, G., et al (1992) Prospective versus retrospective methods of identifying patients with delirium. Journal of the American Geriatric Society, 40, 316-319.

Lewis, L. M., Miller, D. K., Morley, J. E., et al (1995) Unrecognized delirium in emergency department geriatric patients. American Journal Emergency Medicine, 13, 142145

Lindesay, J. (1999) The concept of delirium. Dementia and Geriatric Cognitive Disorders, 10, 310-315.

*Meagher, D. J. (2001). Delirium: optimising management. $B M J, 322,144-149$.

* — \& Trzepacz, P. T. (2000) Motoric subtypes of delirium. Seminars in Clinical Neuropsychiatry, 5, 76-86.

-, O'Hanlon, D., O'Mahony, E., et al (1996) Use of environmental strategies and psychotropic medication in the management of delirium. British Journal of Psychiatry, 168, 512-515

Nakamura, J., Uchimura, S., Yamada, S., et al (1997) Does plasma free-3-methoxy-4-hydroxyphenyl(ethylene)glycol increase in the delirious state? A comparison of the effects of mianserin and haloperidol on delirium. International Clinical Psychopharmacology, 12, 147-152.

Nicholas, L. M. \& Lindsay, B. A. (1995) Delirium presenting with symptoms of depression. Psychosomatics, 36, 471-479.

O'Keeffe, S. T. (1994) Rating the severity of delirium: the Delirium Assessment Scale. International Journal of Geriatric Psychiatry, 9, 551-556.

— \& Gosney, M. (1997) Assessing attentiveness in older hospital patients: global assessment versus tests of attention. Journal of the American Geriatrics Society, 45, 470-473.
Platt, M. M., Breitbart, W., Smith, M., et al (1994) Efficacy of neuroleptics for hypoactive delirium. Journal of Neuropsychiatry and Clinical Neurosciences, 6, 66-67.

Robertsson, B. (1999) Assessment scales in delirium. Dementia and Geriatric Cognitive Disorders, 10, 368-379.

${ }^{*}$ Rockwood, K. (1999). Educational interventions in delirium. Dementia and Geriatric Cognitive Disorders, 10, 426-429.

- Cosway, S., Stolee, P., et al (1994) Increasing the recognition of delirium in elderly patients. Journal of the American Geriatrics Society, 42, 252-256.

Rutherford, L. E., Sessler, C. N., Levenson, J. L., et al (1991) Prospective evaluation of delirium and agitation in a medical intensive care unit. Critical Care Medicine, 19, S81.

Schofield, I. (1997) A small exploratory study of the reaction of older people to an episode of delirium. Journal of Advanced Nursing, 25, 942-952.

Sirois, F. (1988) Delirium: 100 cases. Canadian Journal of Psychiatry, 33, 375-378.

Trzepacz, P. T., Brenner, R., Coffman, G., et al (1988a) Delirium in liver transplant candidates: discriminant analysis of multiple test variables. Biological Psychiatry, 24, 3-14

—, Baker, R. W., Greenhouse, J. (1988b) A symptom rating scale for delirium. Psychiatry Research, 23, 89-97.

-, Mulsant, B. H., Dew, M. A., et al (1998) Is delirium different when it occurs in dementia? A study using the delirium rating scale. Journal of Neuropsychiatry and Clinical Neurosciences, 10, 199-204.

- Mittal, D., Torres, R., et al (2000) Validation of the Delirium Rating Scale-Revised-98 (DRS-R-98). Journal of Neuropsychiatry and Clinical Neurosciences, 12, 156.

Wada, Y. \& Yamaguchi, N. (1993) Delirium in the elderly: relationship of clinical symptoms to outcome. Dementia, 4, 113-116.

Wahlund, L. A. \& Bjorlin, G. A. (1999) Delirium in clinical practice: experiences from a specialized delirium ward Dementia and Geriatric Cognitive Disorders, 10, 389-392.

World Health Organization (1992) The ICD-10 Classification of Mental and Behavioural Disorders. Geneva: WHO.

*indicates articles of particular interest

\section{Multiple choice questions}

\section{Delirium:}

a typically includes a broad range of neuropsychiatric symptoms

b has disturbances of attention as a central feature

c can be diagnosed by the presence of clouding of consciousness alone

$\mathrm{d}$ rarely involves mood disturbances

e is a subtype of acute confusion.

2. In the detection of delirium:

a non-identification is rarely a problem in clinical practice

b up to two-thirds of cases are superimposed on dementia

c detection can be enhanced by routine cognitive assessment of all patients

$\mathrm{d}$ prevalence has decreased in recent years

e misdiagnosis as dementia is rare. 
3. Hypoactive or quiet delirium:

$\mathrm{a}$ is frequently missed in clinical practice

b has a better prognosis than agitated or hyperactive delirium

c does not respond to antipsychotic agents

$\mathrm{d}$ frequently makes patients unrousable

e rarely includes delusions or hallucinations.

4. In the management of patients with delirium:

a iatrogenic causes of delirium are common

b involvement of relatives is generally discouraged

c risk factor reduction allows episode prevention

d delirious patients should not contribute to treatment decisions

e the effectiveness of antipsychotics is principally due to sedative actions.

5. In delirium assessment:

a delirium rating scales allow distinction of delirium from dementia b delirium cannot be accurately assessed in mute patients

c the CAM has good coverage of delirium symptoms

$\mathrm{d}$ the DRS has good coverage of delirium symptoms

e the MMSE has good coverage of delirium symptoms.
MCQ answers

$\begin{array}{llllll}1 & 2 & 3 & 4 & 5\end{array}$

$\begin{array}{llllllll}\text { a } T & \text { a } F & \text { a } T & \text { a } & T & \text { a } T\end{array}$

$\begin{array}{lllllll}b & T & b & T & b & F & b\end{array} \quad$ b $F$

$\begin{array}{lllllllllll}\text { c } & F & \text { c } & T & \text { c } & F & \text { c } & T & \text { c } & F\end{array}$

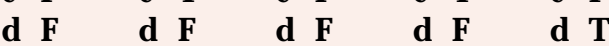

e $\begin{array}{llllllllll} & \text { e } & F & \text { e } & F & \text { e } & F & \text { e } & & \end{array}$

\section{Commentary}

\section{Alastair Macdonald}

Dr Meagher's measured account of delirium, and his proposal for a greater role for psychiatry, brings to mind Jim Birley's extension, when President of the Royal College of Psychiatrists, of Desmond Curran's "Psychiatry Ltd" (Curran, 1952). This tried to redress the overweening presumption that psychiatrists should not only opine on every aspect of medical, social and political life, but also demand hegemony over them. Sadly, for instance, in the case of violent behaviour by people with mental illness, his caution went unheeded, and the results are there for all to see. It is against his injunction that I test the role of psychiatry in delirium.
A distinction must be made between a description (what is the current role of psychiatry - service, research and teaching - in delirium?) and prescription (what should be the role of psychiatry?). Another point is that 'psychiatry' cannot have a role - only psychiatrists can, and then only in a particular, local matrix of service organisation and delivery. I take this matrix from the UK, at this juncture, although of course we arrogate evidence where we will. I will also say nothing further about delirium in childhood; a fascinating yet grossly underresearched topic.

First, description. In terms of service, we need to know where delirious people are, who is dealing

Alastair Macdonald is Professor of Old Age Psychiatry at King's College London (Academic Department, Ladywell Unit, Lewisham Hospital, London SE13 6LH), and Honorary Consultant Psychiatrist in the Mental Health for Older Adults Service of the South London \& Maudsley NHS Trust. His research interests include routine clinical outcomes measurement, delirium, nursing home care for dementia and information systems. 